\title{
Erratum to: Electronic parameters for charge transfer along DNA
}

\author{
L.G.D. Hawke ${ }^{1}$, G. Kalosakas ${ }^{1, a}$, and C. Simserides ${ }^{2}$ \\ 1 Materials Science Department, University of Patras, GR-26504 Rio, Greece \\ 2 Physics Department, University of Athens, GR-15784 Athens, Greece
}

Original article: Eur. Phys. J. E 32, 291 (2010), DOI: 10.1140/epje/i2010-10650-y

Received 8 September 2011

Published online: 28 October 2011 - (c) EDP Sciences / Società Italiana di Fisica / Springer-Verlag 2011

In our discussion of electronic parameters for charge (hole or electron) transfer along DNA we have omitted to mention that, regarding the tight-binding description of hole transport, the corresponding tight-binding parameters should be taken with the opposite sign of the calculated on-site energies and transfer hopping integrals. This means that for describing hole transport at the base-pair level, the on-site energies $E_{\mathrm{H}}^{b p}$ presented in the second row of table 2 and the hopping transfer integrals $t_{\mathrm{H}}^{b p}$ presented in the second column of table 3 should be used with opposite signs in order to provide the tight-binding parameters of eq. (10). Similarly, for describing hole transport at the single-base level, the on-site energies $E_{\mathrm{H}}^{b}$ presented in the eleventh row of table 1 and the hopping transfer integrals $t_{\mathrm{H}}^{b}$ presented in the second column of tables 4-7 should be used with opposite signs in order to provide the tight-binding parameters of eq. (13).

Moreover, on p. 300, 8 lines below eq. (14), in the calculation of charge transfer hopping parameters the separation between adjacent base-pairs in B-DNA should read $3.4 \AA$, instead of $3.14 \AA$.

\footnotetext{
a e-mail: georgek@upatras.gr
} 